Draft VERSion JANUARY 19, 2021

Typeset using LATEX twocolumn style in AASTeX63

\title{
The X-ray pulsar XTE J1858+034 observed with NuSTAR and Fermi/GBM: spectral and timing characterization plus a cyclotron line
}

\author{
C. Malacaria,${ }^{1,2}$ P. Kretschmar ${ }^{3}$ K.K. Madsen, ${ }^{4,5}$ C.A. Wilson-Hodge, ${ }^{6}$ Joel B. Coley ${ }^{7,8}$ P. Jenke, ${ }^{9}$ \\ Alexander A. Lutovinov, ${ }^{10}$ K. Pottschmidt, ${ }^{8,5}$ Sergey S. Tsygankov, ${ }^{11,10}$ And J. Wilms ${ }^{12}$ \\ ${ }^{1}$ NASA Marshall Space Flight Center, NSSTC, 320 Sparkman Drive, Huntsville, AL 35805, USA* \\ ${ }^{2}$ Universities Space Research Association, Science and Technology Institute, 320 Sparkman Drive, Huntsville, AL 35805, USA \\ ${ }^{3}$ European Space Agency (ESA), European Space Astronomy Centre (ESAC), Camino Bajo del Castillo s/n, 28692 Villanueva de la \\ Cañada, Madrid, Spain \\ ${ }^{4}$ CRESST and X-ray Astrophysics Laboratory, NASA Goddard Space Flight Center, Greenbelt, MD 20771, USA \\ ${ }^{5}$ Department of Physics and Center for Space Science and Technology, University of Maryland, Baltimore County, Baltimore, MD 21250 , \\ USA \\ ${ }^{6}$ ST 12 Astrophysics Branch, NASA Marshall Space Flight Center, Huntsville, AL 35812, USA \\ ${ }^{7}$ Department of Physics and Astronomy, Howard University, Washington, DC 20059, USA \\ ${ }^{8}$ CRESST and Astroparticle Physics Laboratory, NASA Goddard Space Flight Center, Greenbelt, MD 20771, USA \\ ${ }^{9}$ University of Alabama in Huntsville (UAH), Center for Space Plasma and Aeronomic Research (CSPAR), 301 Sparkman Drive, \\ Huntsville, Alabama 35899 \\ ${ }^{10}$ Space Research Institute of the Russian Academy of Sciences, Profsoyuznaya Str. 84/32, Moscow 117997, Russia \\ ${ }^{11}$ Department of Physics and Astronomy, FI-20014 University of Turku, Finland \\ ${ }^{12}$ Remeis-Observatory and Erlangen Centre for Astroparticle Physics, Friedrich-Alexander-Universität Erlangen-Nürnberg, \\ Sternwartstr. 7, 96049 Bamberg, Germany
}

\begin{abstract}
Accreting X-ray pulsars (XRPs) undergo luminous X-ray outbursts during which the spectral and timing behavior of the neutron star can be studied in detail. We analyze a NuSTAR observation of the XRP XTE J1858+034 during its outburst in 2019. The spectrum is fit with a phenomenological, a semi-empirical and a physical spectral model. A candidate cyclotron line is found at $48 \mathrm{keV}$, implying a magnetic field of $5.4 \times 10^{12} \mathrm{G}$ at the site of emission. This is also supported by the physical best-fit model. We propose an orbital period of about 81 days based on the visual inspection of the $\mathrm{X}$ ray outbursts recurrence time. Based on Fermi Gamma-ray Burst Monitor data, the standard disk accretion-torque theory allowed us to infer a distance of $10.9 \pm 1.0 \mathrm{kpc}$. Pulse profiles are single-peaked and show a pulsed fraction that is strongly energy-dependent at least up to $40 \mathrm{keV}$.
\end{abstract}

Keywords: X-ray binary stars - stars: neutron - pulsars: individual: XTE J1858+034 - accretion, accretion disks - magnetic fields

\section{INTRODUCTION}

Accreting X-ray pulsars (XRPs) are binary systems consisting of a neutron star (NS) that accretes matter originating from a donor companion star via stellar wind or Roche-lobe overflow. XTE J1858+034 is an XRP discovered with the Rossi X-ray Timing Explorer (RXTE) in 1998 by Remillard et al. (1998) and Takeshima et al. (1998). Those observations also detected X-ray pulsations with a period of $\sim 221 \mathrm{~s}$. X-ray emission from this source has been detected only in a

Corresponding author: cmalacaria@usra.edu

* NASA Postdoctoral Fellow few short outbursts (Nakajima et al. 2019, and references therein), thus preventing to obtain an orbital solution or an in-depth characterization of the system. A Cyclotron Resonant Scattering Feature (CRSF) also was not observed from this source so far. When observed, the energy $E_{\text {cyc }}$ of the fundamental CRSF probes the magnetic field strength at the site of spectral emission, $E_{\text {cyc }} \sim 11.6 \times B_{12}\left(1+z_{\mathrm{g}}\right)^{-1} \mathrm{keV}$, where $B_{12}$ is the magnetic field in units of $10^{12} \mathrm{G}$, and $z_{\mathrm{g}}$ is the gravitational redshift (see Staubert et al. 2019 for a recent review). However, Paul \& Rao (1998) estimated a magnetic field strength of $0.8 \times 10^{12} \times d_{k p c} \mathrm{G}$ (with $d_{k p c}$ the distance value in units of $\mathrm{kpc}$ ), based on the observation of quasiperiodical oscillations in this system. 


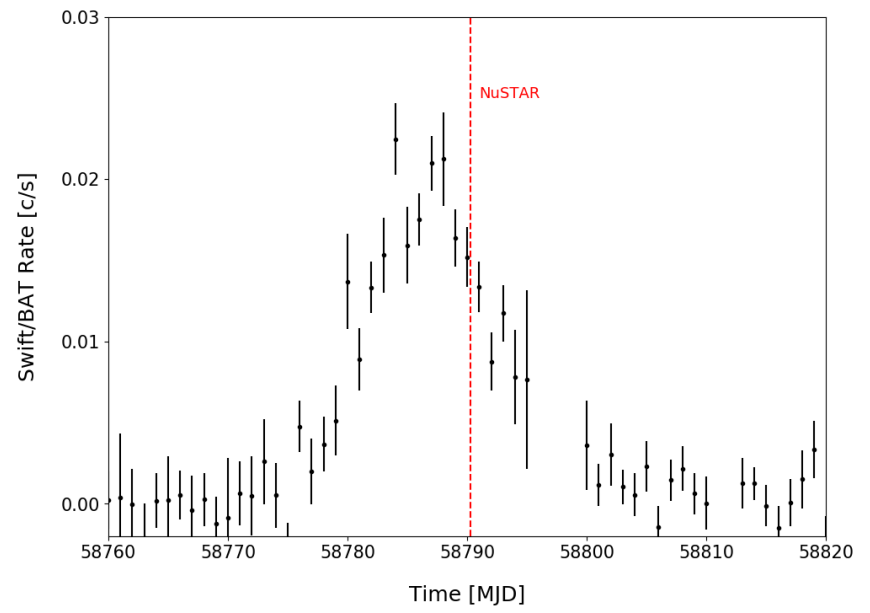

Figure 1. Swift/BAT daily average light curve of XTE J1858+034 during the outburst in 2019 (black dots). The NuSTAR observation time is also shown (red dashed line).

Reig et al. (2004, 2005) proposed a Be-type star for the optical counterpart, of which neither the spectral subtype nor the distance was found. This star was the only one within the hard X-ray error circle from INTEGRAL observations (Molkov et al. 2004) showing $\mathrm{H} \alpha$ emission and was thus proposed as counterpart although it lay outside the error circle of the JEM-X soft X-ray instrument. At an angular offset of 3.5" from the nominal X-ray source position, Gaia found an optical candidate counterpart (Bailer-Jones et al. 2018), mentioned in Malacaria et al. (2020) as possible counterpart, but likely unassociated given the large offset. In addition, the Gaia counterpart is at an angular offset of $103^{\prime \prime}$ from, and thus clearly not associated with, the optical counterpart proposed by Reig et al. (2005). This question is discussed in more detail in the accompanying paper by Tsygankov et al. (in press), who identify a counterpart based on Chandra and ground telescope observations, with a probable distance of $7-14 \mathrm{kpc}$.

Recently, the source has undergone a new outburst episode (Nakajima et al. 2019), and was observed with NuSTAR. Here we study its spectral and timing characteristics and finally form a consistent general overview for the X-ray behaviour of XTE J1858+034, including a distance estimate based on accretion-torque theory. The analysis presented here is complemented by the work in the accompanying paper by Tsygankov et al. (in press).

\section{DATA REDUCTION}

NuSTAR (Harrison et al. 2013) was launched in 2012. It is currently the only X-ray mission with a telescope able to focus hard X-rays above $10 \mathrm{keV}$. NuSTAR consists of two identical co-aligned telescopes that focus Xray photons onto two independent Focal Plane Modules,
FPMA and FPMB. At the focus of each telescope module are four $(2 \times 2)$ solid-state cadmium zinc telluride (CdZnTe) imaging detectors. These provide wide-band (3-79 keV) energy coverage with a FWHM of $18^{\prime \prime}$ and a spectral resolution of $400 \mathrm{eV}$ at $10 \mathrm{keV}$.

NuSTAR observed XTE J1858+034 on 2019 November 3 (ObsID 90501348002, MJD 58790), during an outburst (see Fig. 1). The total exposure time was about 44 ks. NuSTAR data were reduced with NUSTARDAS v1.9.5 provided by the HEASOFT v6.27.2 and using the $C A L D B$ 20200526 (Madsen et al. 2020). Cleaned events were obtained following the standard NuSTAR guidelines. The resulting images are shown in Fig. 2. Source spectra were extracted through the NUPRODUCTS routine. The source extraction region was a $65^{\prime \prime}$ radius circular region centered on the source, while the background was extracted from a source-free region on the same detector with radii of $90^{\prime \prime}$ and $105^{\prime \prime}$ for FPMA and FPMB, respectively. We also verified that shifting the extraction regions in order to account for the offset between the images from the two modules does not significantly affect the results. However, in FPMB part of the source events fall on the chip gap between detectors 0 and 3 , resulting in unaccounted loss of effective area. Moreover, $N u S$ $T A R$ detectors suffer from absorption due to a CdZnTe dead layer and a Pt coating at the top of the detectors, an effect that is calibrated through observations of the Crab in stray light mode (Madsen et al. 2017). However, the absorption curve can be degenerate with other effective area effects, and for detector 3 this has caused part of the detector-related absorption to be included in the vignetting curve, thus resulting in spectral differences in the low energy spectrum when compared to detector 0 (priv. comm. with the NuSTAR Science Operations Team). All these factors led us to exclude the entire detector 3 from the FPMB source extraction region. We obtained the FPMB ARF from a $15^{\prime \prime}$ radius circular region centered on the source, which ensures that the detector absorption of detector 3 did not get included. Similarly, to avoid accidentally including the RMF from detector 3 during RMF generation the RMF for detector 0 was obtained directly from the CALDB (nuBcutdet0_20100101v001).

Spectral data were analyzed using XSPEC v12.11.01 (Arnaud 1996). NuSTAR data were used in the range $3-60 \mathrm{keV}(3.5-60 \mathrm{keV}$ for FPMB to further enhance consistency between spectra in the lowest channels), above which the background dominates. Spectra were rebinned to have at least 50 counts per bin.

\section{RESULTS}

\subsection{Spectral analysis}



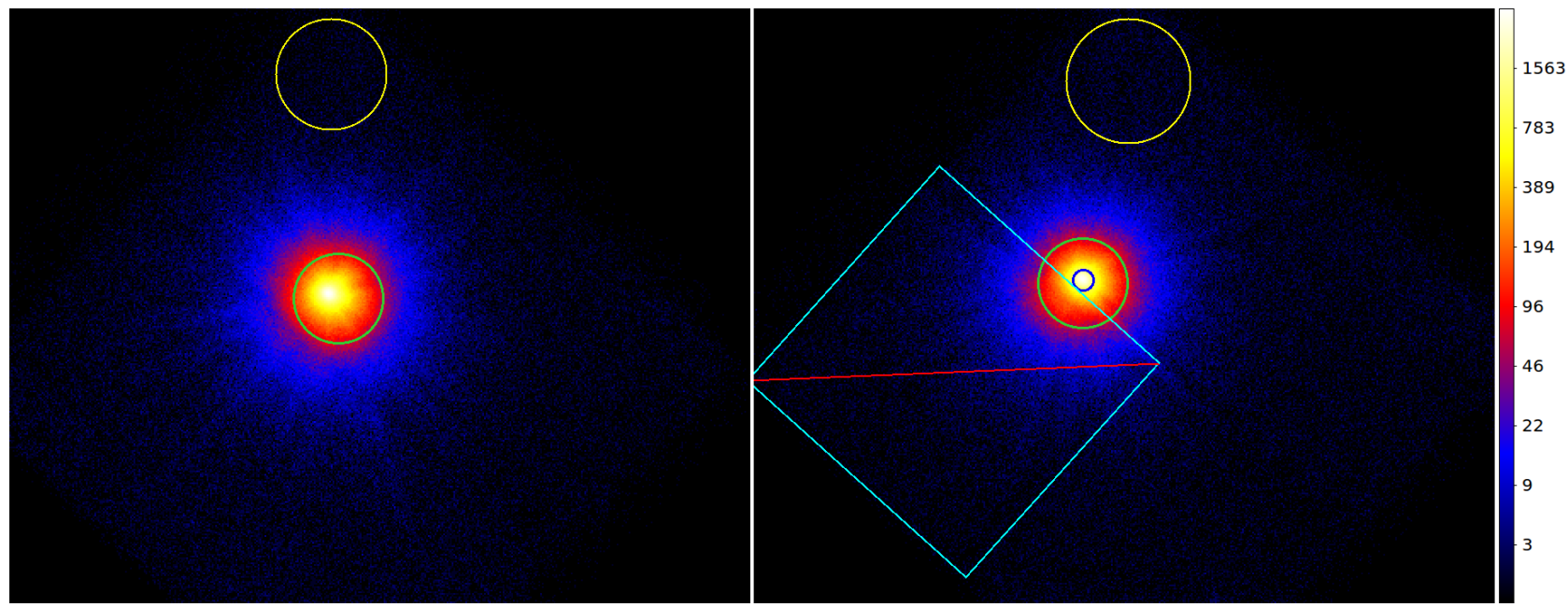

Figure 2. NuSTAR images of XTE J1858+034 as observed in November 2019 from FPMA (left) and FPMB (right). Circular green regions centered on the source represent the source extraction regions. Yellow solid circles on the top corner represent the background extraction regions. For FPMB, the blue small circle represents the ARF extraction region, while the barred cyan square represents the exclusion of detector 3 (see text). The color bar shows the number of counts per pixel.

XTE J1858+034 as observed by NuSTAR in November 2019 clearly shows a hard spectrum. FPMA and FPMB spectra have been fitted simultaneously, allowing for a cross-normalization factor. Although the crossnormalization factor between FPMA and FPMB is usually of the order of a few percent (Madsen et al. 2015), the limited ARF extraction region adopted in our analysis for FPMB (see Sect. 2) is expected to reduce the cross-normalization value significantly. For the spectral fit, standard phenomenological and semi-empirical continuum models have been employed, namely two variants of the cutoff power-law model (cutoffpl and highecut*pow in XSPEC) and a Comptonization model of soft photons in a hot plasma (compTT in XSPEC, Titarchuk 1994), respectively. To obtain an acceptable fit, the cutoffpl and highecut*pow models need an additional component in the lower energy band, which has been modeled as a blackbody emission as found in other accreting XRPs (see, e.g., La Palombara \& Mereghetti 2006). However, the blackbody temperature is high with respect to other XRPs, indicating that the phenomenological model is likely inadequate. Moreover, we also tested a purely-physical model of thermal and bulk Comptonization of the seed photons produced by cyclotron cooling (Ferrigno et al. 2009, bwcycl in XSPEC). For a fixed value of mass and radius of the accreting NS, the bwcycl model has six free parameters, namely the accretion rate $\dot{M}$, the magnetic field strength $B$, the accretion column radius $r_{0}$, the electron temperature $T_{e}$, the photon diffusion parameter $\xi$ and the Comptonization parameter $\delta$. This model was successfully used to fit the broad-band energy spectrum of a number of bright
( $1^{37} \mathrm{erg} \mathrm{s}^{-1}$ ) accreting XRPs (see, e.g., Epili et al. 2017; D'Aì et al. 2017; Wolff et al. 2016).

For all tested models, the photoelectric absorption component and elemental abundances were set according to Wilms et al. 2000 (tbabs in XSPEC) to account for photoelectric absorption by neutral interstellar matter (or column density $N_{H}$ ), and assuming model-relative (wilm) solar abundances. Given that the Galactic $N_{H}$ in the direction of the source is about $1.7 \times 10^{22} \mathrm{~cm}^{-2}$ (HI4PI Collaboration et al. 2016), all models show important local absorption values. All tested models also were equipped with a Gaussian emission line at $6.4 \mathrm{keV}$ to account for the $\mathrm{Fe} \mathrm{K} \alpha$ fluorescence emission.

All fit continuum models show absorption-like residuals in the range $40-50 \mathrm{keV}$. These residuals can be modeled with a Gaussian absorption line (see Fig. 3). The improvement in the best-fit statistics is maximum in the compTT model, i.e. $\Delta \chi^{2}=878$. Other models show an improvement of $\Delta \chi^{2} \gtrsim 400$, with the lowest $\Delta \chi^{2}$ derived from the highecut*pow model. The significance of the line in the compTT model has been assessed through Monte Carlo simulations. For this task, the XSPEC simftest routine was adopted, which allows to simulate a chosen number of spectra based on the actual data and test the resulting $\Delta \chi^{2}$ between each instance fit when the additional model component (the Gaussian absorption line in our case) is included. Following Bhalerao et al. (2015); Bodaghee et al. (2016), the column density parameter was fixed to its best-fit value, and the energy and width of the Gaussian absorption line were left free to vary within their $90 \%$ confidence region in order to improve the speed and convergence of the fits. Simulations results are reported in Fig. 4 


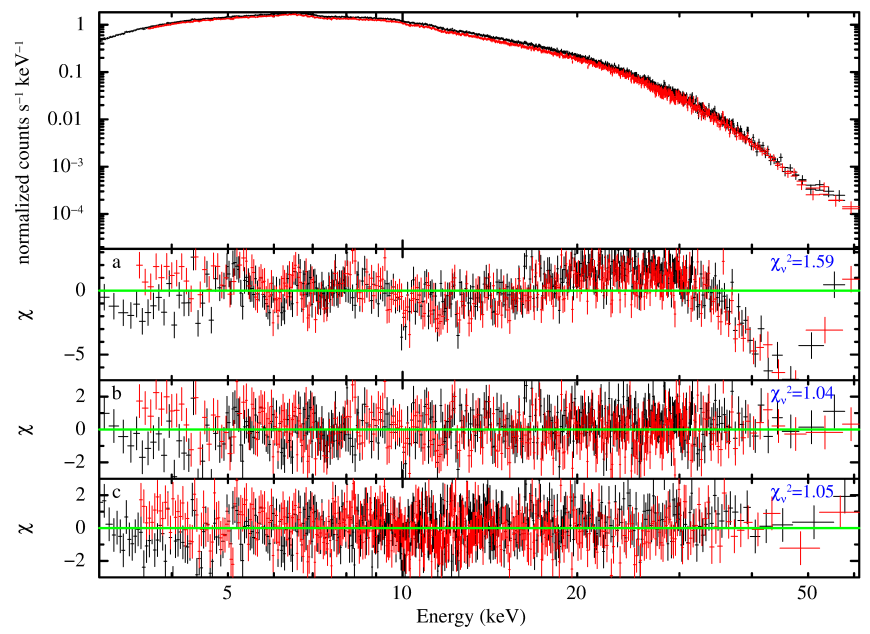

Figure 3. Top: XTE J1858+034 spectrum as observed by $N u S T A R$ in 2019 and fit with a ComptTT model. Lower panels are referred with a letter in the upper left corner. Panel $a$ : residuals of the ComptTT model. Panel $b$ : residuals of the best-fit ComptTT model including a Gaussian absorption line at $\sim 48 \mathrm{keV}$ (see Table 1). Panel $c$ : residuals of the bestfit BWCYC IIa model including a Gaussian absorption line at $\sim 48 \mathrm{keV}$ (see Table 2). Spectra and residuals have been rebinned for plotting purpose. The blue text in the right corners of the lower panels shows the correspondent model $\chi^{2}$ divided by $\nu$ degrees of freedom.

for a $10^{4}$ iterations process and confirm the significance of the absorption feature at $>3 \sigma$ c.l. Following MarcuCheatham et al. (2015), we also investigated the impact of a variable background normalization on the absorption feature parameters. Using the XSPEC tool recorn, it was found that the absorption line parameters do not change significantly if the normalization of the background spectrum is increased up to a $50 \%$ higher level, thus strengthening the interpretation of the absorption feature as real and not due to artifacts. The feature was also observed in phase-resolved spectra presented in the accompanying paper by Tsygankov et al. (in press). Interpreting the feature at $48 \mathrm{keV}$ as a CRSF, and assuming a gravitational redshift of $z_{g}=0.3$ (for NS mass and radius of $1.4 M_{\odot}$ and $10 \mathrm{~km}$, respectively), a magnetic field strength of $\mathrm{B}=(5.4 \pm 0.1) \times 10^{12} \mathrm{G}$ is obtained.

Following the bwcycl model instructions ${ }^{1}$, it is convenient to freeze some of the model parameters in order to improve the computational speed and help the fit converge to the best-fit parameters. Once the best-fit was found, the column density $N_{H}$ also was fixed to its best-fit value to help the fit converge and to obtain parameters errors. Mass and radius of the NS were fixed

\footnotetext{
${ }^{1}$ https://heasarc.gsfc.nasa.gov/xanadu/xspec/manual/node148. html.
}

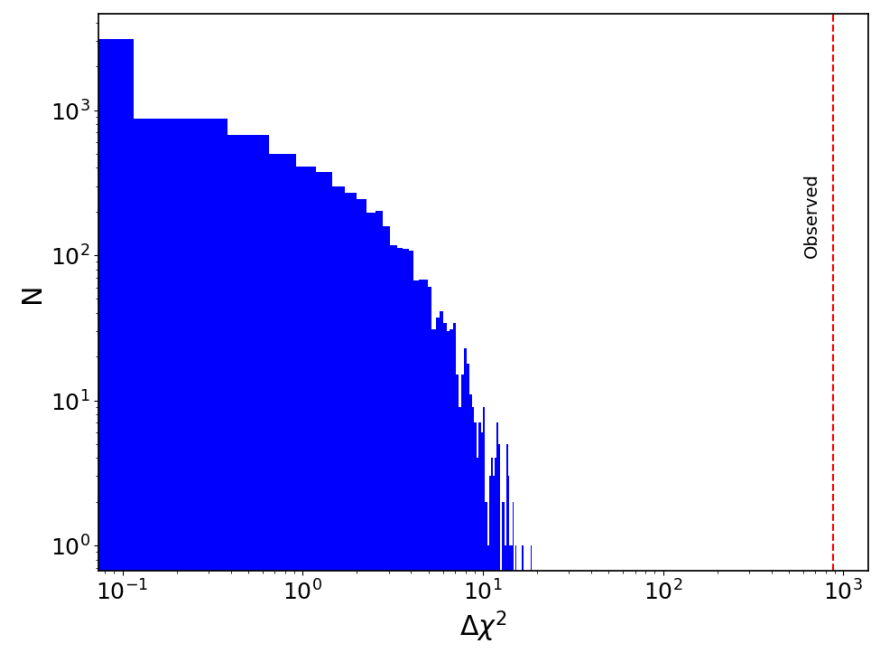

Figure 4. Results of 10000 Monte Carlo simulations to test the significance of the Gaussian absorption line in the CompTT model. The solid histogram shows the frequency (y-axis) of $\Delta \chi^{2}$ values (x-axis) obtained in the simulation. The red dashed line shows the observed $\Delta \chi^{2}=878$.

to their canonical values of $1.4 \mathrm{M}_{\odot}$ and $10 \mathrm{~km}$, respectively. However, it is preferable to also fix the values of the NS magnetic field, its distance and its mass accretion rate (as derived by the observed luminosity). For XTE J1858+034, there are no previous conclusive estimations of the magnetic field, while a measurement of the distance is necessary for the latter two parameters. As mentioned in Sect. 1, the closest Gaia counterpart to the nominal X-ray position found by Molkov et al. (2004) was unlikely associated with the X-ray source or with the optical counterpart proposed by Reig et al. (2005). This was ascertained by Tsygankov et al. (in press), who shown that either of those possible counterparts is consistent with the much better constrained $\mathrm{X}$-ray source location available through new Chandra observations. However, the distance to the X-ray system had not been estimated before their work, which in any case did not constrain it very much. Therefore, we opted for a different approach to obtain a more stringent value of the distance, based on the spin-up $(\dot{P})$ measured by Fermi-GBM.

To this aim, the publicly available spin-frequency values from GBM were used ${ }^{2}$. The spin-up was measured during an interval of about 6 days around MJD 58786 . The resulting spin-up value is $|\dot{P}|=10.5421(6) \mathrm{s} \mathrm{yr}^{-1}$ (see also Malacaria et al. 2020, and references therein). Since the orbital parameters of this system are un-

\footnotetext{
2 https://gammaray.nsstc.nasa.gov/gbm/science/pulsars/ lightcurves/xtej1858.html
} 
Table 1. Best-fit results of XTE J1858+034 spectral analysis with a cutoff power-law model cutoffpl and a Comptonization model CompTT. All reported errors are at $1 \sigma$ c.l.

\begin{tabular}{|c|c|c|}
\hline & cutoffpl & CompTT \\
\hline $\mathrm{C}_{F P M B}$ & $0.747_{-0.001}^{+0.001}$ & $0.747_{-0.001}^{+0.001}$ \\
\hline $\mathrm{N}_{\mathrm{H}}\left[10^{22} \mathrm{~cm}^{-2}\right]$ & $7.6_{-0.4}^{+0.4}$ & $5.8_{-0.3}^{+0.3}$ \\
\hline $\mathrm{kT}_{b b}[\mathrm{keV}]$ & $5.2_{-0.1}^{+0.2}$ & - \\
\hline $\operatorname{norm}_{b b}$ & $0.0152_{-0.0007}^{+0.0002}$ & - \\
\hline $\mathrm{E}_{K \alpha}[\mathrm{keV}]$ & $6.47_{-0.02}^{+0.02}$ & $6.48_{-0.02}^{+0.02}$ \\
\hline$\sigma_{K \alpha}[\mathrm{keV}]$ & $0.26_{-0.02}^{+0.02}$ & $0.28_{-0.02}^{+0.02}$ \\
\hline $\operatorname{norm}_{K \alpha}\left(10^{-4}\right)$ & $5.5_{-0.4}^{+0.4}$ & $5.9_{-0.4}^{+0.4}$ \\
\hline$\Gamma$ & $0.03_{-0.22}^{+0.29}$ & - \\
\hline HighECut [keV] & $3.5_{-0.5}^{+1.5}$ & - \\
\hline $\operatorname{norm}_{\Gamma}{ }^{*}$ & $0.025_{-0.004}^{+0.006}$ & - \\
\hline $\mathrm{T} 0[\mathrm{keV}]$ & - & $1.02_{-0.02}^{+0.02}$ \\
\hline $\mathrm{kT}_{C o m p T T}[\mathrm{keV}]$ & - & $5.61_{-0.04}^{+0.05}$ \\
\hline$\tau_{p}[\mathrm{keV}]$ & - & $7.07_{-0.06}^{+0.06}$ \\
\hline $\operatorname{norm}_{C o m p T T}$ & - & $0.0228_{-0.0003}^{+0.0003}$ \\
\hline $\mathrm{E}_{\text {gabs }}[\mathrm{keV}]$ & $46.8_{-0.8}^{+1.0}$ & $48.0_{-0.7}^{+0.8}$ \\
\hline$\sigma_{g a b s}[\mathrm{keV}]$ & $7.7_{-0.7}^{+1.0}$ & $8.6_{-0.5}^{+0.6}$ \\
\hline Strengthgabs & $14.9_{-2.6}^{+5.1}$ & $21.3_{-2.5}^{+2.0}$ \\
\hline Flux $^{\dagger}$ & $1.499_{-0.003}^{+0.003}$ & $1.499_{-0.003}^{+0.003}$ \\
\hline$\chi^{2} /$ d.o.f. & $1645 / 1574$ & $1643 / 1573$ \\
\hline
\end{tabular}

* In units of photons $/ \mathrm{keV} / \mathrm{cm}^{2} / \mathrm{s}$ at $1 \mathrm{keV}$. † Flux calculated for the entire model in the $3-60 \mathrm{keV}$ band and reported in units of $10^{-9} \mathrm{erg} \mathrm{cm}^{-2} \mathrm{~s}^{-1}$. Flux values with estimated errors were derived using the cflux model from XSPEC as resulting from FPMA.

known, we tested the contribution of orbital modulation to the observed spin-up. First, although Doroshenko et al. (2008) report a possible orbital period for this source of about 380 days, we notice that its significance is low, while a visual inspection of the Swift/BAT (Krimm et al. 2013) data ${ }^{3}$ for this source revealed an outbursts recurrence of $\sim 81$ days (see Fig. 5), here assumed as the orbital period. Moreover, for a Kor M-type optical companion star (Tsygankov et al., in press), we adopted a value of the mass function $f(M)=M_{*}{ }^{3} \sin ^{3} i /\left(M_{*}+M_{N S}\right)^{2}=1$, where $M_{*}$ and $M_{N S}$ are the mass of the companion and that of the NS, respectively, $i$ is the binary system inclination. This corresponds to a value of the semi-major projected axis, $a_{x} \sin i \simeq 190 \mathrm{l}$-s. Also, the data required only a small value of the eccentricity, assumed here as $e \simeq 0.1$. Fi-

\footnotetext{
3 https://swift.gsfc.nasa.gov/results/transients/weak/ XTEJ1858p034/
}

Table 2. Best-fit results of XTE J1858+034 spectral analysis with different configurations of the physical Bulk+Thermal Comptonization model bwcyc. All sets have the distance value fixed at $d=10.9 \mathrm{kpc}$. All reported errors are at $1 \sigma$ c.l.

\begin{tabular}{|c|c|c|c|}
\hline & BWCYCa & $\mathrm{BWCYCb}$ & BWCYCc \\
\hline $\mathrm{C}_{F P M B}$ & $0.748_{-0.001}^{+0.001}$ & $0.748_{-0.001}^{+0.001}$ & $0.748_{-0.001}^{+0.001}$ \\
\hline $\mathrm{N}_{\mathrm{H}}\left[10^{22} \mathrm{~cm}^{-2}\right]$ & $8.6_{-0.2}^{+0.2}$ & $8.4_{-0.2}^{+0.2}$ & $8.4_{-0.8}^{+0.3}$ \\
\hline $\mathrm{E}_{K \alpha}[\mathrm{keV}]$ & $6.47_{-0.02}^{+0.02}$ & $6.48_{-0.02}^{+0.02}$ & $6.47_{-0.02}^{+0.8}$ \\
\hline$\sigma_{K \alpha}[\mathrm{keV}]$ & $0.27_{-0.02}^{+0.02}$ & $0.28_{-0.02}^{+0.02}$ & $0.27_{-0.02}^{+0.02}$ \\
\hline $\operatorname{norm}_{K \alpha}\left(10^{-4}\right)$ & $5.5_{-0.4}^{+0.4}$ & $5.6_{-0.3}^{+0.3}$ & $5.6_{-0.4}^{+0.4}$ \\
\hline$\xi$ & $4.2_{-1.7}^{+0.7}$ & $3.2_{-0.8}^{+0.8}$ & $2.9_{-0.6}^{+1.0}$ \\
\hline$\delta$ & $0.4_{-0.1}^{+0.3}$ & $0.6_{-0.1}^{+0.3}$ & $0.7_{-0.2}^{+0.3}$ \\
\hline $\mathrm{B}\left[10^{12} \mathrm{G}\right]$ & $4.4_{-0.3}^{+0.1}$ & 5.4 (fixed) & 5.4 (fixed) \\
\hline$\dot{M}\left[10^{17} \mathrm{~g} / \mathrm{s}\right]$ & 1.2 (fixed) & 1.2 (fixed) & $1.1_{-0.1}^{+0.1}$ \\
\hline $\mathrm{T}_{e}[\mathrm{keV}]$ & $5.6_{-0.6}^{+0.2}$ & $5.5_{-0.5}^{+0.1}$ & $5.2_{-0.4}^{+0.2}$ \\
\hline $\mathrm{r}_{0}[\mathrm{~m}]$ & $73_{-22}^{+9}$ & $66_{-12}^{+22}$ & $49_{-10}^{+29}$ \\
\hline $\mathrm{d}[\mathrm{kpc}]$ & 10.9 (fixed) & 10.9 (fixed) & 10.9 (fixed) \\
\hline $\mathrm{E}_{\text {gabs }}[\mathrm{keV}]$ & $48.3_{-0.7}^{+0.7}$ & $48.6_{-1.3}^{+0.5}$ & $48.3_{-1.1}^{+1.0}$ \\
\hline$\sigma_{\text {gabs }}[\mathrm{keV}]$ & $10.3_{-2.1}^{+0.7}$ & $9.6_{-1.2}^{+1.2}$ & $9.3_{-0.6}^{+1.1}$ \\
\hline Strength & $25.4_{-6.4}^{+4.2}$ & $27.6_{-4.2}^{+3.1}$ & $\begin{array}{l}-0.6 \\
25.3_{-3.9}^{+4.0}\end{array}$ \\
\hline Flux $^{\dagger}$ & $1.499_{-0.003}^{+0.003}$ & $1.499_{-0.002}^{+0.003}$ & $1.499_{-0.003}^{+0.003}$ \\
\hline$\chi^{2} /$ d.o.f. & $1648 / 1574$ & $1648 / 1575$ & $1648 / 1574$ \\
\hline
\end{tabular}

${ }^{*}$ unconstrained. ${ }^{\dagger}$ Flux calculated in the $3-60 \mathrm{keV}$ band and reported in units of $10^{-9} \mathrm{erg} \mathrm{cm}^{-2} \mathrm{~s}^{-1}$. Flux values with estimated errors were derived using the cflux model from XSPEC as calculated for FPMA.

nally, an epoch of $T 0=53436$ MJD was chosen at the beginning of the first of the recurring outbursts. An argument of periapse of $\omega=107^{\circ}$ was found to best-fit the GBM frequency values assuming no accretion torque. This orbit assumed, the maximum orbital contribution to the spin-up was found to be only about $10 \%$.

Assuming a magnetic field strength of $5.4 \times 10^{12} \mathrm{G}$ and adopting the NuSTAR measured flux of $1.5 \times 10^{-9} \mathrm{erg} \mathrm{cm}^{-2} \mathrm{~s}^{-1}$ (see Table 1 and 2), the standard accretion-disk torque theory (Ghosh \& Lamb 1979) can be used to infer the distance of the source according to the equation:

$$
\begin{array}{r}
-\dot{P}=5 \times 10^{-5} \mathrm{~s} \mathrm{yr}^{-1} M_{1.4}^{-3 / 7} R_{6}^{6 / 7} I_{45}^{-1} \\
\times n\left(\omega_{s}\right) \mu_{30}^{2 / 7}\left(P L_{37}^{3 / 7}\right)^{2}
\end{array}
$$

where $M_{1.4}$ is the NS mass in units of $1.4 \mathrm{M}_{\odot}, R_{6}$ is the NS radius in units of $10^{6} \mathrm{~cm}, I_{45}$ is the moment of inertia in units of $10^{45} \mathrm{~g} \mathrm{~cm}^{2}, \mu_{30}$ is the magnetic moment in units of $10^{30} \mathrm{G} \mathrm{cm}^{3}, n\left(\omega_{s}\right) \approx 1.4$ is the dimensionless torque, $P$ is the spin period in seconds and $L_{37}$ is the bolometric luminosity in units of $10^{37} \mathrm{erg} \mathrm{s}^{-1}$. For 


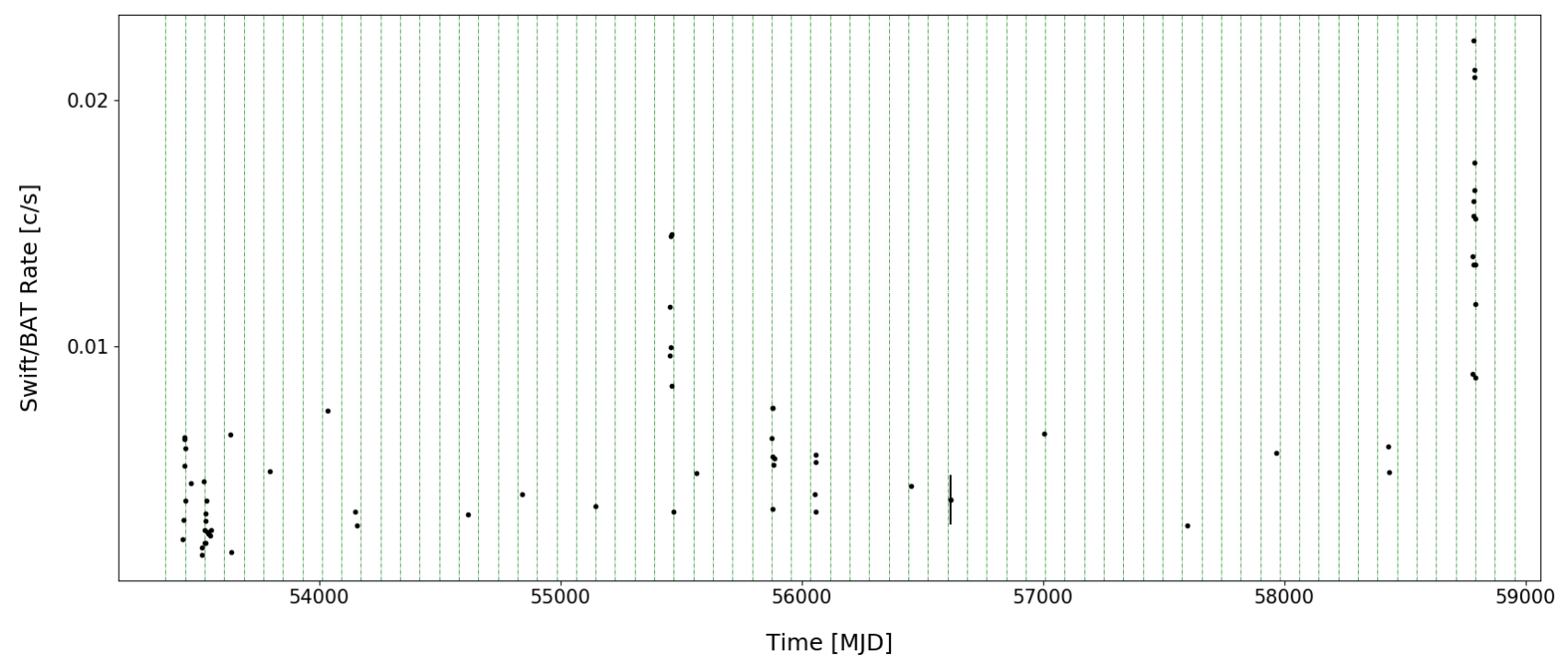

Figure 5. Swift/BAT light curve of XTE J1858+034. Only data points more significant than $3 \sigma$ are reported. A typical error bar is shown only for one data point around MJD 56500 for clarity. Green dash-dotted lines are separated by 81 days.

a measured $|\dot{P}|=10.5 \mathrm{syr}^{-1}$, Eq. 1 allows to infer a distance of $d=10.9 \pm 1.0 \mathrm{kpc}$ (estimated uncertainty at $1 \sigma$ c.l.). This distance value is also independently confirmed by the analysis of the optical companion star as reported in the accompanying paper by Tsygankov et al. (in press), and it was used to characterize different configurations of the bwcyc model. The corresponding mass accretion rate $\dot{M}=1.2 \times 10^{17} \mathrm{~g} \mathrm{~s}^{-1}$ was adopted altogether, derived assuming a luminosity $L=\eta \dot{M} c^{2}$, with efficiency $\eta=0.2$ (Sibgatullin \& Sunyaev 2000). The different configurations of the tested model are reported in Table 2 include a set with the magnetic field strength as a free parameter (BWCYCa), one with the magnetic field strength fixed to $5.4 \times 10^{12} \mathrm{G}$ (BWCYCb), and one with a free $\dot{M}$ and a fixed magnetic field strength (BWCYCc).

\subsection{Timing analysis}

For the timing analysis, the nuproducts task was used to obtain light curves out of calibrated and cleaned events. These light curves were corrected for livetime, exposure and vignetting effects, and were extracted in the following energy bands: $3-10,10-20,20-30$, $30-40,40-60$ and $3-60 \mathrm{keV}$.

All light curves were barycentered using the barycorr tool and the NuSTAR clock correction file nuCclock20100101v103. The light curve in the $3-$ $60 \mathrm{keV}$ energy band was binned to $5 \mathrm{~s}$ and used to search for pulsations around the known 221 s periodicity with the epoch folding method (Leahy 1987). The procedure results in a measured period of $P=218.393(2) \mathrm{s}$. Pulsations are significant at $>99 \%$. The uncertainty was estimated by simulating 500 light curves based on real data and altered with Poisson noise.

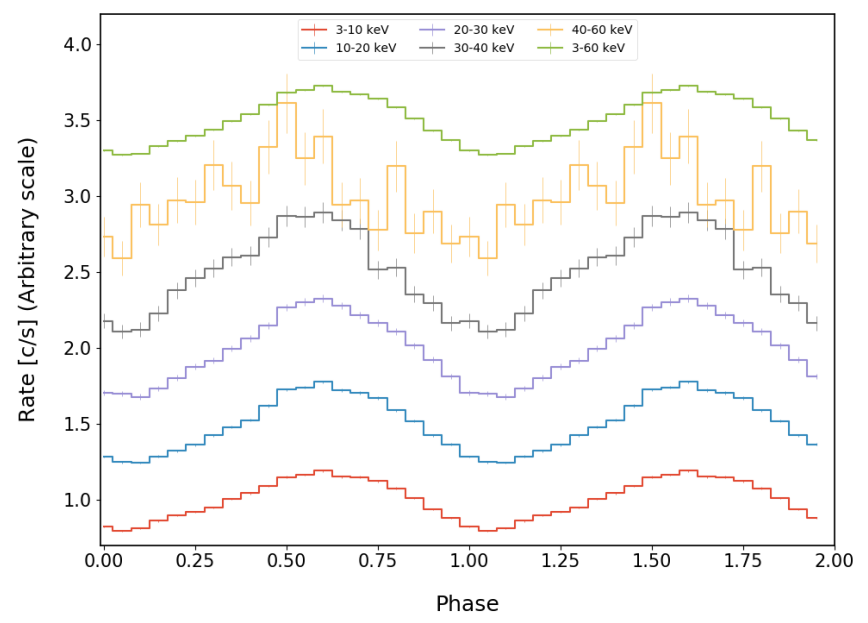

Figure 6. XTE J1858+034 energy-resolved pulse profiles as observed by $N u S T A R$. The energy band increases upwards. Pulse profiles are shown twice in phase and rescaled in count rate for clarity.

Light curves in different energy bands were folded to the best-fit spin period to obtain pulse profiles with a resolution of 20 phase bins (see Fig. 6). In turn, these were used to explore the pulsed fraction variation as a function of the energy (see Fig. 7). The pulsed fraction here is defined as $\left(I_{\max }-I_{\min }\right) /\left(I_{\max }+I_{\min }\right)$, where $I_{\max }, I_{\min }$ are the maximum and minimum pulse profile count rate, respectively.

\section{DISCUSSION}

4.1. Thermal Comptonization and a candidate cyclotron line 
The hard spectrum of XTE J1858+034 resembles that of other accreting X-ray pulsars observed both at low and high luminosity and well fit by a CompTT model (Mukerjee et al. 2020, and references therein). The observation of CompTT spectra in accreting X-ray pulsars is usually interpreted as the result of thermal Comptonization processes in which the thermal energy of the accreting gas is transferred to the seed photons originating from the NS hotspots (Becker \& Wolff 2007). An increasing number of these sources also show that an additional CompTT component emerges in the highenergy range of the spectrum at low-luminosity stages (Tsygankov et al. 2019a,b). Although the formation of such component is not clear yet, it is likely due to a combination of cyclotron emission and following thermal Comptonized emission from a thin overheated layer of the NS atmosphere (see Tsygankov et al. 2019b, and references therein). In this context, X Persei is a remarkable case since it has been shown that the cyclotron line in its spectrum can be mimicked by the convolution of the two CompTT spectral components around the energy where the flux from the low- and high-energy components is comparable (Doroshenko et al. 2012). However, among the sources whose spectrum is formed by two CompTT components, X Persei is the one with the highest electron temperature of the hard-energy CompTT component, $k T \sim 15 \mathrm{keV}$. If the absorption feature at $\sim 48 \mathrm{keV}$ in XTE J1858+034 is in fact resulting from the blend of two CompTT components, the high-energy CompTT would peak around $22 \mathrm{keV}$. This would make XTE J1858+034 the most extreme among the X-ray pulsars that show such spectral shape. However, such a spectral shape has so far only been observed in low-luminosity X-ray pulsars, while our analysis (see Sect. 3.1) show that the source is located at a relatively large distance of $10.9 \mathrm{kpc}$, thus implying a high-luminosity source (also supported by the analysis of Tsygankov et al., in press). A second Gaussian or CompTT component that peaked above $45 \mathrm{keV}$ was also tested in place of the absorption feature, but could not be successfully fit $\left(\chi_{\text {red }}^{2}>1.4\right)$, although possibly due to the lack of statistics above $60 \mathrm{keV}$.

\subsection{Thermal and bulk Comptonization}

With the newly inferred source distance value of $d=$ $10.9 \mathrm{kpc}$, the derived flux of $1.5 \times 10^{-9} \mathrm{erg} \mathrm{cm}^{-2} \mathrm{~s}^{-1} \mathrm{im}-$ plies a luminosity of $2.1 \times 10^{37} \mathrm{erg} \mathrm{s}^{-1}$. Adopting this distance value, all different sets of the bwcyc model are statistically equivalent and all parameters show acceptable values. For the BWCYCa configuration, the returned magnetic field strength of $4.2 \times 10^{12} \mathrm{G}$ is consistent within $2 \sigma$ with that inferred from the candidate CRSF. Notably, the BWCYCb model with fixed values of the distance, mag-

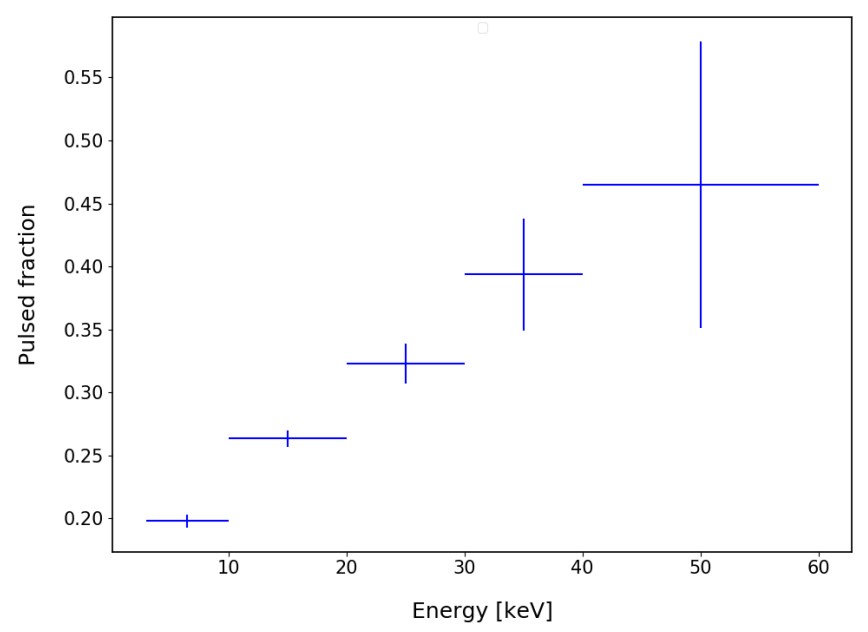

Figure 7. XTE J1858+034 pulsed fraction as a function of the energy during the NuSTAR observation in November 2019 .

netic field strength and accretion rate also fits the data and returns acceptable values of the best-fit parameters. The BWCYCc fit returns the smallest (but still acceptable) $r_{0}$ value, and a mass accretion rate $\dot{M}$ that is almost coincident with that inferred from the X-ray (isotropic) luminosity.

In any case, when interpreting the results from the bwcyc model, it is important to keep in mind that, as reported in Ferrigno et al. (2009), the BWcyc model may need adjustments in the spectral parameters with respect to the original prescriptions. For example, the best-fit magnetic field value may differ from that inferred by the CRSF if the spectrum is formed at a NS site that is spatially different than the CRSF forming region. Likewise, the best-fit mass accretion rate $\dot{M}$ can be different from that inferred by the X-ray luminosity due to an uncertain efficiency conversion factor $(\eta)$ and anisotropic emission.

\subsection{Timing results}

Pulse profiles of XTE J1858+034 as observed by NuSTAR show a single-peak structure and a shape that is only weakly energy-dependent (see Fig. 6). This is typically observed at low mass accretion rates (see, e.g. Malacaria et al. 2015), and qualitatively interpreted as the beaming pattern resulting from a pencil-beam emission. However, single-peaked pulse profiles are also observed at high accretion rates, like in the case of Pulsating Ultra-Luminous X-ray sources (PULXs), e.g. Swift J0243.6+6124, where single-peak pulse profiles persist at high luminosity and only switch to more complex profiles at super-Eddington luminosity (WilsonHodge et al. 2018). 
The pulsed fraction shows a considerable energydependence, and almost doubles from $20 \%$ in the $3-$ $10 \mathrm{keV}$ to about $40 \%$ in the $30-40 \mathrm{keV}$ energy band, above which the lack of statistics prevent us from drawing firm conclusions.

\section{CONCLUSIONS}

We analyzed the NuSTAR observation of the 2019 outburst of the XRB XTE J1858+034. The source, relatively poorly studied, has now been characterized in multiple ways. A candidate cyclotron line is found in its spectrum at $48 \mathrm{keV}$. This implies a magnetic field strength of $5.4 \times 10^{12} \mathrm{G}$, consistent with the value obtained from the physical fitting model of thermal and bulk Comptonization bwcyc in its best-fit configurations. We propose an orbital period of about 81 days based on the visual inspection of the X-ray outbursts recurrence time. Arguments are given to review the previously proposed optical counterpart and its distance value in favor of a distance of $10.9 \pm 1.0 \mathrm{kpc}$ obtained from standard accretion-torque theory.

\section{ACKNOWLEDGMENTS}

This research has made use of data and software provided by the High Energy Astrophysics Science Archive Research Center (HEASARC), which is a service of the Astrophysics Science Division at NASA/GSFC and the High Energy Astrophysics Division of the Smithsonian Astrophysical Observatory. We acknowledge extensive use of the NASA Abstract Database Service (ADS). C.M. is supported by an appointment to the NASA Postdoctoral Program at the Marshall Space Flight Center, administered by Universities Space Research Association under contract with NASA. AAL and SST acknowledge support from the Russian Science Foundation (grant 19-12-00423).

\section{REFERENCES}

Arnaud, K. A. 1996, in Astronomical Society of the Pacific Conference Series, Vol. 101, Astronomical Data Analysis Software and Systems V, ed. G. H. Jacoby \& J. Barnes, 17

Bailer-Jones, C. A. L., Rybizki, J., Fouesneau, M., Mantelet, G., \& Andrae, R. 2018, AJ, 156, 58

Becker, P. A., \& Wolff, M. T. 2007, ApJ, 654, 435

Bhalerao, V., Romano, P., Tomsick, J., et al. 2015, MNRAS, 447, 2274

Bodaghee, A., Tomsick, J. A., Fornasini, F. M., et al. 2016, ApJ, 823, 146

D'Aì, A., Cusumano, G., Del Santo, M., La Parola, V., \& Segreto, A. 2017, MNRAS, 470, 2457

Doroshenko, V., Santangelo, A., Kreykenbohm, I., \&

Doroshenko, R. 2012, A\&A, 540, L1

Doroshenko, V. A., Doroshenko, R. F., Postnov, K. A., Cherepashchuk, A. M., \& Tsygankov, S. S. 2008, Astronomy Reports, 52, 138

Epili, P., Naik, S., Jaisawal, G. K., \& Gupta, S. 2017, MNRAS, 472, 3455

Ferrigno, C., Becker, P. A., Segreto, A., Mineo, T., \& Santangelo, A. 2009, A\&A, 498, 825

Ghosh, P., \& Lamb, F. K. 1979, ApJ, 234, 296

Harrison, F. A., Craig, W. W., Christensen, F. E., et al. 2013, ApJ, 770, 103

HI4PI Collaboration, Ben Bekhti, N., Flöer, L., et al. 2016, A\&A, 594, A116
Krimm, H. A., Holland, S. T., Corbet, R. H. D., et al. 2013, ApJS, 209, 14

La Palombara, N., \& Mereghetti, S. 2006, A\&A, 455, 283

Leahy, D. A. 1987, A\&A, 180, 275

Madsen, K. K., Forster, K., Grefenstette, B. W., Harrison, F. A., \& Stern, D. 2017, The Astrophysical Journal, 841, 56

Madsen, K. K., Grefenstette, B. W., Pike, S., et al. 2020, arXiv e-prints, arXiv:2005.00569

Madsen, K. K., Harrison, F. A., Markwardt, C. B., et al. 2015, ApJS, 220, 8

Malacaria, C., Jenke, P., Roberts, O. J., et al. 2020, ApJ, 896,90

Malacaria, C., Klochkov, D., Santangelo, A., \& Staubert, R. 2015, A\&A, 581, A121

Marcu-Cheatham, D. M., Pottschmidt, K., Kühnel, M., et al. 2015, ApJ, 815, 44

Molkov, S. V., Cherepashchuk, A. M., Revnivtsev, M. G., et al. 2004, ATel, 274, 0

Mukerjee, K., Antia, H. M., \& Katoch, T. 2020, arXiv e-prints, arXiv:2005.14044

Nakajima, M., Negoro, H., Kurogi, K., et al. 2019, The Astronomer's Telegram, 13217, 1

Paul, B., \& Rao, A. R. 1998, A\&A, 337, 815

Reig, P., Kougentakis, T., \& Papamastorakis, G. 2004, ATel, 308, 0

Reig, P., Negueruela, I., Papamastorakis, G., Manousakis, A., \& Kougentakis, T. 2005, A\&A, 440, 637 
Remillard, R., Levine, A., Takeshima, T., et al. 1998, IAUC, 6826, 0

Sibgatullin, N. R., \& Sunyaev, R. A. 2000, Astronomy Letters, 26, 699

Staubert, R., Trümper, J., Kendziorra, E., et al. 2019, A\&A, 622, A61

Takeshima, T., Corbet, R. H. D., Marshall, F. E., Swank, J., \& Chakrabarty, D. 1998, IAUC, 6826, 0

Titarchuk, L. 1994, ApJ, 434, 570
Tsygankov, S. S., Doroshenko, V., Mushtukov, A. e. A., et al. 2019a, MNRAS, 487, L30

Tsygankov, S. S., Rouco Escorial, A., Suleimanov, V. F., et al. 2019b, MNRAS, 483, L144

Wilms, J., Allen, A., \& McCray, R. 2000, ApJ, 542, 914

Wilson-Hodge, C. A., Malacaria, C., Jenke, P. A., et al. 2018, ApJ, 863, 9

Wolff, M. T., Becker, P. A., Gottlieb, A. M., et al. 2016, ApJ, 831, 194 